\title{
DESIGN OF BIOMECHANICAL SENSOR BASED LOWER LIMBS REHABILITATION TRAINING MACHINE
}

\author{
Hui Shen \\ Department of Physical Education, Jinling Institute of Technology, Jiangning University Town, \\ Nanjing, Jiangsu, 211169, China \\ huis69@126.com
}

\begin{abstract}
With the increase of patients who have difficulty in moving by lower limbs in recent years, how to make lower limbs recover effectively has attracted more and more attentions. Most of the patients are unable to do rehabilitation training independently. Therefore, robot technology is gradually used to design lower limbs training machine in medical field. Biomechanical sensor is efficient in collecting the gait information of patients because of its characteristics such as favorable dynamic performance and small volume. This study designed a lower limbs rehabilitation training machine based on biomechanical sensor and verified the practicality of the sensor in lower limbs rehabilitation training machine, which provides a reference and suggestion for studies on the development of rehabilitation machine.
\end{abstract}

Keywords: Gait Rehabilitation; Biomechanical Sensor; Rehabilitation Training Machine.

\section{Introduction}

With the development of society, the sales volume of cars improves greatly. As a result, the occurrence of traffic accidents causes a large number of fractured and paralysed patients. Patients developing movement disorder become more and more, and the incidence among young people becomes higher and higher. A study [14] suggested that, reasonable and effective lower limbs training of lower limbs could produce a powerful effect on the repair of movement function. The shortcomings of artificial rehabilitation training such as low efficiency and high cost severely affect the rehabilitation condition of those patients [5].

Therefore, studying a kind of intelligentized rehabilitation training machine and formulating a set of new rehabilitation training plan have been extensively concerned by the society. Wang et al. [12] established a kinematic mathematical model for passive training and proposed a rigid connecting rod model based on the full analysis of lower limbs movement. Human rehabilitation training machine technology which belongs to the category of artificial intelligence system and involves the content of multiple fields such as biomechanics, electronics, medicine and mechanics has developed rapidly in recent years and is a huge breakthrough in medical field [1]. To evaluate the rehabilitation training efficacy of patients with injured lower limbs, gait data acquisition and analysis are needed. Based on biomechanics, this study acquired and analyzed lower limbs gait parameters using sensors. A targeted lower limbs rehabilitation training machine was designed to achieve better man-machine conversation after the collected data were analyzed in details. The machine could collect the actions of operators through inductors and analyze whether those actions satisfied health standards; if not, then the actions will be adjusted to achieve the purpose of lower limbs rehabilitation training.

\section{Theory}

\section{Sensors}

In the field of sports biomechanics, exact values are required in the analysis and study of actions. Exact values are usually obtained by transforming nonelectric physical quantity such as force, speed and accelerated speed to electric parameters according to certain technology. Mechanical sensors and speed sensors are needed in the conversion between the two parameters [7]. In this study, the gait of patients during rehabilitation training was recorded and analyzed using pressure sensors and acceleration speed sensors to help patients do training better and recover rapidly.

\section{Gait analysis}

Gait analysis refers to measure and quantitatively estimate the skeleton and muscle of patients during exercise during lower limbs rehabilitation training and evaluate whether the walking state of a patient is healthy based on the principles of biomechanics [4]. Gait analysis is usually applied in exercise rehabilitation medicine as an effective approach for disease diagnosis and rehabilitation assessment [2]. It can assist determining whether surgery is 
successful or not and the efficacy of rehabilitation treatment.

\section{Design Scheme of Lower Limbs Training Machine}

The structure of lower limbs rehabilitation training machine

Wearable lower limbs rehabilitation training machine is a complex mechanical system. To sense gait information and correct inaccurate gait information, the perceptual system, control system and drive executive system of the lower limbs rehabilitation training machine should coordinate together [3]. The perceptual system of the lower limbs rehabilitation training machine is an important component in the whole mechanical system, which is composed of pressure sensors and acceleration sensors and is responsible for collecting information such as plantar pressure, joint angle and body acceleration [10]. Based on the information, the moving posture of human body can be known. Then the robot can be controlled to help patients fulfill rehabilitation training. The structure of the lower limbs rehabilitation training machine is shown in Figure 1.

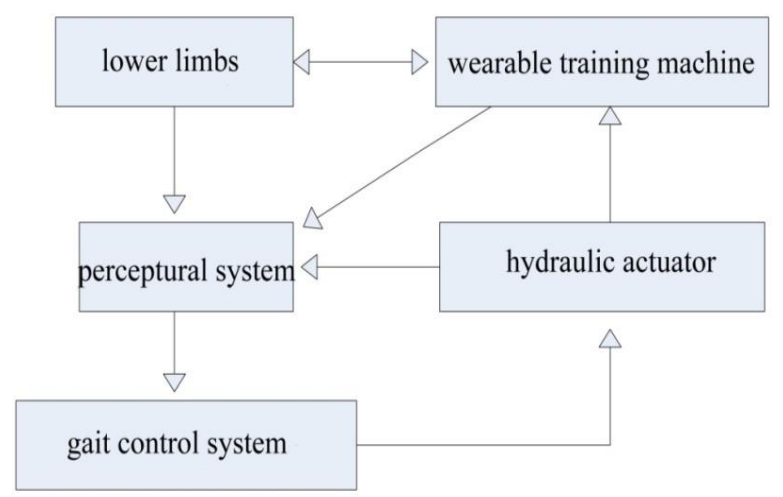

Figure 1: The structure of the lower limbs rehabilitation training machine

\section{Selection of Plantar Pressure Sensor}

Plantar ground reaction force is important gait information which can be used in the analysis and identification of movement of human body. The main work of the perceptual system in the lower limbs rehabilitation training machine was analyzing the changes of plantar pressure. There are several requirements for the real-time detection of plantar pressure using plantar pressure sensor during walking. The installation of pressure sensors should not affect the normal walking of human; the measurement rage of sensors should be large enough to satisfy the demands of people with different weights; sensors should have favorable time lag property and repeatability to ensure the accuracy of plantar measurement system.
In view of the above points, Polyvinylidene Fluoride (PVDF) piezoelectric sensor was selected. PVDF is a novel sensing material of high-molecular polymer type [13]. Compared to the traditional piezoelectric materials, PVDF sensor has favorable linearity, sensitivity of mechano-electric conversion and repeatability. Moreover, it is featured by quick response, wide frequency response, large dynamic range, easy matching of acoustic impedance, strong mechanical proper, light weight, good softness, small thickness, impact resistance, low risks of water and chemical drugs pollution and good ductility; hence it is a good choice as the material for piezoelectric sensors.

Shape design, structure processing and stickup are three procedures for the manufacture of a mechanical sensor using PVDF piezoelectric thin film. In the process of shape design, sensors in circular structure can satisfy different requirements. Therefore, both PVDF thin film and substrate holder were made into circular shape. During structure processing, PV membrane was used in cantilever beam, and the substrate holder was made using conductive silicone sheet; then stamping was performed with a cylindrical mold ( $=10 \mathrm{~mm}$ ). As to sticking technology, conductive mercury glue.

After manufacture of the piezoelectric sensor, a metal clamp was designed. The head of the sensor was inserted to the metal clamp to make stress even. To effectively collect gait information of the patients, each shoe was installed with three sensors, one at heel, one at the second phalanx, and one at the fifth phalanx. Figure 2 demonstrated the positions of the pressure sensors at shoe sole.

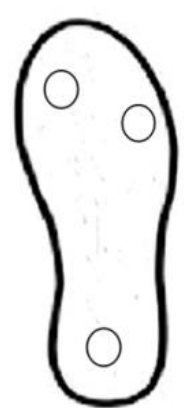

Figure 2: The positions of the pressure sensors at shoe sole

\section{Selection of Acceleration Sensors}

Acceleration acquisition system is mainly composed of upper computer, main data acquisition system and data acquisition subsystem [6]. Digital triaxial accelerometer ADXL345 which was developed by ADI Company using Micro-electromechanical Systems (MEMS) technology was applied as the acceleration sensor. It could timely collect the acceleration values of joints and then send them to the main system. 
The acceleration information of human body movement was collected by the acceleration sensor. The acceleration information at the horizontal axis and numerical axis was regarded as the research subjects. When the patients stood up and sat up, the motion states of human body were upright by body and upright by stick; the angular acceleration angle could be set as $0^{\circ}$. When the acceleration angle was, it could be decomposed to vertical direction and forward and backward directions.

$$
\begin{aligned}
& a_{x}=g \sin \theta \\
& a_{y}=g \cos \theta
\end{aligned}
$$

It could be deduced from formula (1) and (2) that,

$$
\begin{aligned}
& \frac{a_{y}}{a_{x}}=\tan \theta \\
& \theta=\arctan \frac{a_{y}}{a_{x}}
\end{aligned}
$$

\section{Experiment on Lower Limbs Rehabilitation Training Machine}

The lower limbs rehabilitation machine was tested after manufacture. An experimenter was asked to wear the rehabilitation training machine. Firstly, they did the postures of stand up or sit up. After posture determination, the experimenter expressed corresponding action intentions.
They expressed intention of stand up if sitting up or intention of sit up or walking if standing up.

The posture changed after the rehabilitation machine did corresponding actions. The rehabilitation machine could respond through detecting the movement intention of body.

\section{Experimental Results of Plantar Piezoelectric Sensors}

After the above experiment, the plantar pressure at different motion states was collected using the plantar pressure sensor. Figure 3 demonstrates the distribution of plantar pressure of the experimenter in the whole process of walking.

As shown in Figure 3, the peak plantar pressure at the second phalanx was $300 \mathrm{~N}$, the peak plantar pressure of the fifth phalanx (140 N) appeared before that of the second phalanx, and the peak plantar pressure of the heel (90 N) appeared before that of the phalanx.

It could be noted from Figure 3 that, the pressure of heals increased and the pressure of the second phalanx was large when the body was supported by two legs; the pressure of the supporting leg transferred from the heel to the sole when the experiment walked in single leg. Hence it could be concluded that the plantar pressure demonstrated regular distribution in a cycle, which could be used to determine the current motion and predict the following motion posture.

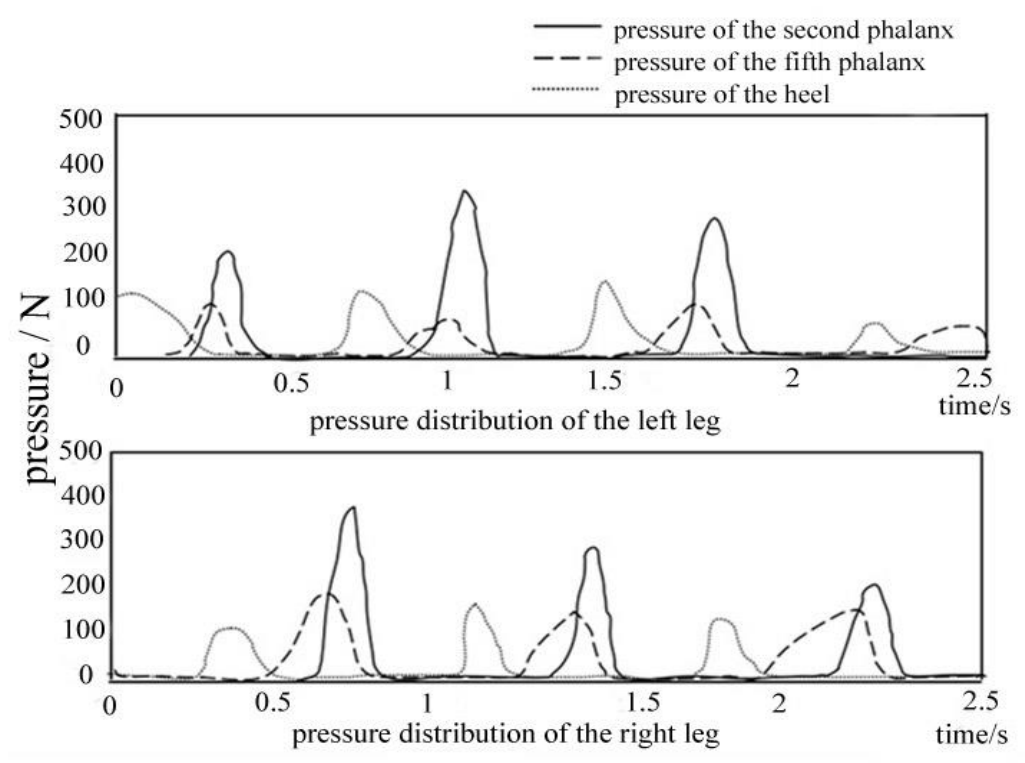

Figure 3: The discussion of plantar pressure of the experimenter in the whole process of walking 


\section{Experimental Results of the Acceleration Sensor}

The information collected by the acceleration sensor was analyzed by Matlab software, and the results are shown in Figure 4.

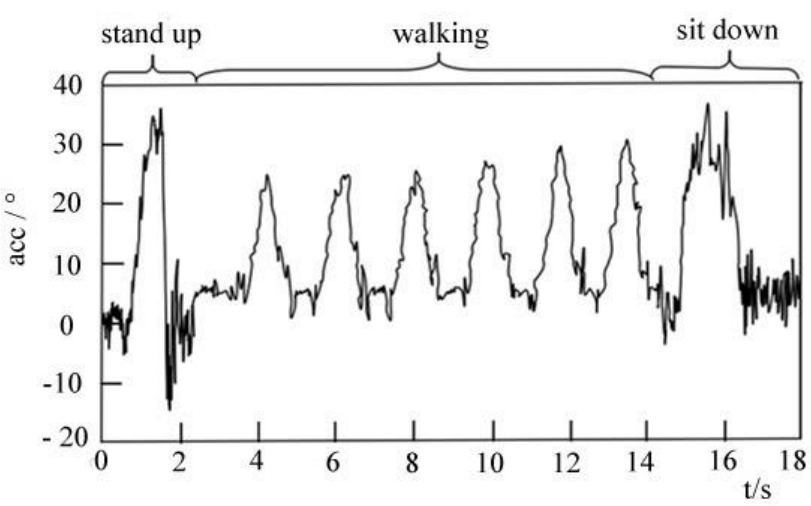

Figure 4: Inclination angle of the back when the experimenter was at different motion states

Considering that the lower limbs of the patient were unable to move in the early stage of rehabilitation, walking stick test was performed. Figure 5
The inclination angle of the back fluctuated in $1 \mathrm{~s}$ when the experimenter sat down, and the peak value was $35^{\circ}$. The walking action lasted for about $2 \mathrm{~s}$; the acceleration angle of the back changed regularly during walking, and the peak value was $23^{\circ}$. demonstrates the changes of acceleration angle of the walking sticks.

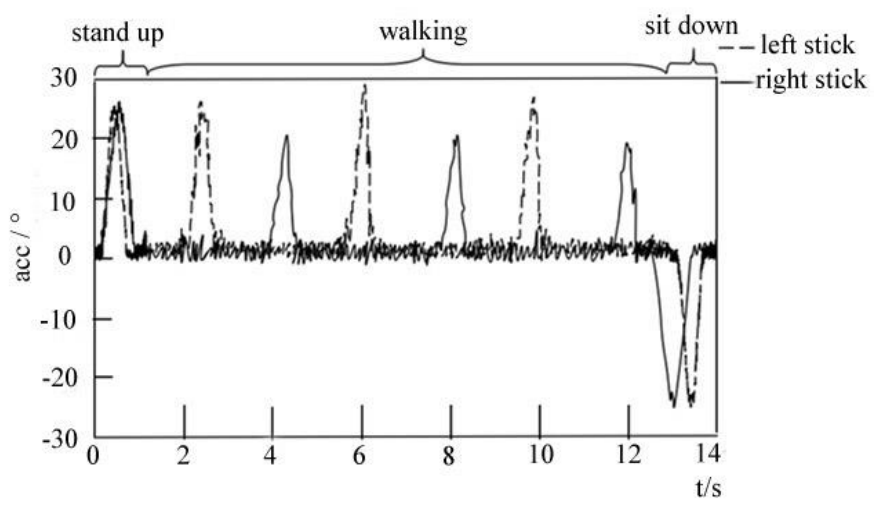

Figure 5 Changes of acceleration angles of the walking sticks

The experimenter held the walking sticks and intended to stand up in the posture of sitting, the angle of the sticks changed simultaneously; the maximum inclination angle of the sticks was $18^{\circ}$. The patient stood up under the assistance of walking sticks, and meanwhile the inclination angle of the sticks changed, and the maximum inclination angle was $23^{\circ}$. During walking, the two walking sticks moved alternately, and the changes of acceleration of the sticks were regular. The peak values of the two sticks were different; the peak value of the left and right stick was $25^{\circ}$ and $19^{\circ}$ respectively.

After the analysis of data obtained in different motion states, it could be concluded that the inclination angle of the back changed with the changes of motion states. The peak value of inclination angle of the back was $33^{\circ}$ when the patient stood up and sat down and $20^{\circ}$ when the patient walked. When the patient had no intention to stand up, the angle might also exceed $20^{\circ}$. Therefore, the angle of the sticks was taken as another determination method. It could be noted from the above content that the acceleration angle of the sticks was $25^{\circ}$ at maximum when the patient was sitting. A proper threshold could be selected as another signal for standing up, and it is also effective as the trigger signal for sitting down.

It could be concluded from Fig. 4 and 5 that inclination angles of the back and sticks could be used for helping rehabilitation training machine 
obtain the motion information of patients when the lower limbs completely lost motion ability but the upper limbs were normal. Digital triaxial accelerometer ADXL345b could be applied to obtain the acceleration information of the back and sticks, and then the acceleration angles of the back and sticks could be calculated. Inclination angles of the back and sticks when patients changed motion posture could be known after data analysis. Then the threshold could be determined; as a result, the perceptual system could accurately determine the motion intention of patients.

\section{Conclusion}

With the development of science and technology, studies on lower limbs rehabilitation training machine become deeper and deeper. Sun et al. [11] fixed an ultrasonic gait detector on a lower limbs rehabilitation machine and determined the gait information of patients based on the instantaneous speed of swing and walking positions. Khoshdel [8] investigated voltage-based adaptive impedance force control for a lower-limb rehabilitation robot to improve training efficiency. This study proposed a biochemical sensor based lower limbs rehabilitation training machine, performed experiments on the training machine, and analyzed the information collected by the biomechanical sensors. The sensors could collect and analyze the information of motion postures of patients and moreover correct incorrect motion postures, which can provide a reference and suggestion for the development of lower limbs rehabilitation training machine in the future.

\section{References}

[1] Byun S. D., T. D. Jung, C. H. Kim, Y. S. Lee. (2011). Effects of the sliding rehabilitation machine on balance and gait in chronic stroke patients a controlled clinical trial. Clin Rehabil, 25(5):40815.

[2] Chirakanphaisarn N. (2014). Measurement and analysis system of the knee joint motion in gait evaluation for rehabilitation medicine. Fourth International Conference on Digital Information and Communication Technology and It's Applications, 315-320.
[3] Chu Y., Y. Shao, L. Chen. (2011). Analysis and design of a wearable robot for lower-limb rehabilitation training. Appl Mechan Mater, 135136(3):569-86.

[4] Gabel M., R. Gilad-Bachrach, E. Renshaw, A. Schuster. (2012). Full body gait analysis with Kinect. Engineering in Medicine \& Biology Society. 2012 Annual International Conference of the IEEE, 1964-1967.

[5] Guo B. J., J. H. Han, X. P. Li, T. T. Fang, A. M. You. (2016). Research and design of a new horizontal lower limb rehabilitation training robot. Int J Adv Robot Syst, 13(1):1.

[6] Herranen H., A. Kuusik, T. Saar, M. Reidla, R. Land, O. Martens, J. Majak. (2014). Acceleration data acquisition and processing system for structural health monitoring. Metrology for Aerospace (MetroAeroSpace), 244-248.

[7] Hood S., T. Mcbain, M. Portas, I. R. Spears. (2012). Measurement in sports biomechanics. Measurem Contr, 45(6):182-186.

[8] Khoshdel V. (2015). Voltage-based adaptive impedance force control for a lower-limb rehabilitation robot. Adv Robot, 29(15):961971.

[9] Lee S. H., A. Goswami. (2010). Ground reaction force control at each foot: A momentum-based humanoid balance controller for non-level and non-stationary ground. IEEE/RSJ International Conference on Intelligent Robots and Systems, 3157-3162.

[10] Meng X. L., Z. Q. Zhang, S. Y. Sun, J. K. Wu, W. C. Wong. (2012). Biomechanical model-based displacement estimation in micro-sensor motion capture. Meas Sci Technol, 23(7):5510155111(11).

[11] Sun B., J. Shen, Q. Zhao, Q. H. Zhang. (2012). Gait detection and analysis based on omnidirectional lower limb rehabilitation robot. International Conference on Automatic Control and Artificial Intelligence, 1102-1105.

[12] Wang H., H. Zhen, Q. Gu, H. Hou, R. Fan. (2013) HAM-based man-machine modeling and kinematic analysis for a lower limb rehabilitation training apparatus. Int J Digit Content Technol Appl, 7(4):462-471.

[13] Wang Y. R., J. M. Zheng, G. Y. Ren, P. H. Zhang, C. $\mathrm{Xu}$. (2011). A flexible piezoelectric force sensor based on PVDF fabrics. Smart Mater Struct, 20(4):045009.

[14] Wu J. W., L.Y. Shen, Y. N. Zhang, J. Q. Qian. (2013). A treadmill speed adaptive control method for lower limb rehabilitation robot. Adv Mater Res, 655-657:1158-1163. 